\title{
Evolutionary Mechanism of Frangibility in Social Consensus System Based on Negative Emotions Spread
}

\author{
Yao-feng Zhang, ${ }^{1,2}$ Hong-ye Duan, ${ }^{2,3}$ and Zhi-lin Geng ${ }^{2}$ \\ ${ }^{1}$ Collaborative Innovation Center of China Pilot Reform Exploration and Assessment-Hubei Sub-Center, \\ Hubei University of Economics, Wuhan 430205, China \\ ${ }^{2}$ School of Information Management and Statistics, Hubei University of Economics, Wuhan 430205, China \\ ${ }^{3}$ Faculty of Mathematics and Statistics, Hubei University, Wuhan 430062, China
}

Correspondence should be addressed to Zhi-lin Geng; zhilingeng@163.com

Received 20 March 2017; Accepted 22 May 2017; Published 28 June 2017

Academic Editor: Pasquale De Meo

Copyright (c) 2017 Yao-feng Zhang et al. This is an open access article distributed under the Creative Commons Attribution License, which permits unrestricted use, distribution, and reproduction in any medium, provided the original work is properly cited.

To study the social consensus system under the spread of negative emotions, the nonlinear emergence model of frangibility of social consensus system is established based on Multiagent method, and effects of emotions spread frequency, opinion leaders, and shielding behavior of government on the frangibility of social consensus system are revealed. The simulation results show that the low-frequency negative emotions spread is better than the high-frequency one for reducing the frangibility of social consensus system. Low-frequency negative emotions spread will lead to the group polarization, while high frequency will lead to the collapse of system. The joining of opinion leaders who are with negative emotions can promote the frangibility of social consensus system, and collapse speed of social consensus system tends to increase with the influence of opinion leaders. Shielding behavior of government cannot effectively block the spread of negative emotions. On the contrary, it will enhance the frangibility of social consensus system.

\section{Introduction}

With the social transformation and the popularity of Internet, people's lives extend onto the Internet from reality gradually. So, the interpersonal relationship networks show complex characteristics of interaction between online and offline. With the fast spread of information on Internet, a complex social consensus system forms. This complex system is not only affected by the individual emotions within the group but also affected by the interference of external events. Through many times' diffusion of negative emotions, imbalance of group memory [1] tends to emerge. Then, collective behaviors on network frequently happen, which may result in many new collective behaviors in reality [2] and eventually lead to fragility of social system. The famous examples include Arab Spring [3] and Occupy Wall Street [4].

The original research of system frangibility began in the study of natural disaster system in late 1960s [5], and it refers to the changes of the system's structures and functions coursed by the sensitivity of the disturbance and lacking of resistance. Most early studies focused on the natural disasters [6], climate changes [7], ecosystem [8], groundwater system [9], and other natural sciences. With the rise of complexity science, studies about the frangibility of social system emerged gradually, like urban system, information system, economic system, and so on [10]. In particular, the frangibility of complex networks has attracted scholars' attention widely [11], examples include the frangibility of email networks [12] and Internet [13] and transport networks and infrastructure networks [14].

Generally, for the studying of system frangibility, there are two kinds of methods. One is the evaluation index system [5, 15], and the other one is mathematical model which develops with the rise of the studies in complex network frangibility [16]. All kinds of comprehensive research methods based on these two methods are fully discussed and applied, such as AHP, entropy method, principal component analysis, Gray Cluster Analysis, fuzzy comprehensive evaluation method, and function method. Though the above research methods have pushed the studies of system frangibility to a new level, 
most of the studies consider the system as a static system with little consideration of the complex structure of the system and seldom consider the coupling interaction between consensus diffusion and the frangibility of social system in the Internet age.

Because the social consensus system is not only a dynamic system with high frequency of update but also an interactive system between virtual world and real world, during the spread of consensus, we could not only take emotions spread as the cause (or effect) of frangibility but also take frangibility as the cause (or effect) of emotions spread. So, the above traditional methods could not reveal the inherent mechanisms of the frangibility evolution from the microlevel. In recent years, scientific computing (including modeling and simulation) has become the third paradigm of scientific research after scientific experiments and theoretical derivation [17]. The Multiagent System [18-21] (MAS) plays an indispensable and important role in complex system study because of its complex features such as dynamic highdimensional, coupling feedback and the overall emergence [22]. Some recent studies show that MAS is becoming an important method in the research of social system frangibility $[23,24]$.

According to the above analysis, we proposed a Multiagent simulation model for frangibility evolution in social consensus system. This model consists of two mechanisms which describe the processes of information spread: emotion diffusion mechanism and emotion influence mechanism. Emotion diffusion mechanism is used to describe the process of group information dissemination in the hot event, and the emotion influence mechanism presents the evolutional process of the internal viewpoints in the group after the information dissemination. We simulate many times occurrences of the negative events in social consensus system by repeating the above two processes serially and explore the influences on frangibility evolution from three factors: event frequency, intervention of opinion leaders, and government shielding behavior. Because many studies show that the interpersonal relationship networks have Free-Scale characteristics [25], we use BA Networks [25] as the topology structure of information spread network of social consensus system in this paper.

Compared with previous studies, this paper has the following differences: at first, most of the previous studies consider the system as a static system or only consider the impact of one node failure on the system frangibility [6$10,12]$. In this paper, we will study the frangibility of dynamic systems under repeated negative emotion diffusion. Secondly, most of the previous simulation studies focus on the cascade failure process of network nodes [23, 24], but the diffusion mechanism and the influence mechanism of node failures are not distinguished. In this paper, the two mechanisms are, respectively, corresponding to two kinds of process, emotion diffusion and emotion influence (postdiffusion evolution), which is much more fitting the actual characteristics of social consensus system. Thirdly, most of the simulations in the past discussed focus on the frangibility of social system caused by emergent events but paid little attention to the emergent events caused by frangibility of social system. In this paper, the interactive phase-disturbing process of the two factors is fully considered and analyzed with specific cases.

The paper is structured as follows. In the next section, we will present the networks model and the Multiagent model for frangibility evolution in social consensus system. Then, we will take "1.25 Incident in Egypt" [3] as an example to analyze the simulation results in Section 3 which we only consider the influences of spread frequency at first and, then, introduce the opinion leaders and shielding behavior of government into the system gradually. Finally, we will draw the conclusions with a brief discussion in the last section.

\section{The Model}

Studies have shown that either in real society or on Internet, interpersonal relationship networks exhibit the characteristic of Free-Scale [25]. Considering that the social consensus transmits through interpersonal relationship networks, we adopt BA Networks proposed by Barabási and Albert as the information spread network in social consensus system [25]. Assuming that the network has only $m_{0}$ nodes in the beginning, a new node with a connection degree $m(m<$ $m_{0}$ ) is added at each time step, and the new node connects to $m$ different nodes already existing in the network. The probability that new node connects with the existing node $i$ is

$$
P_{i}=\frac{k_{j}}{\sum_{j=1}^{N} k_{j}},
$$

where $N$ is the total number of nodes and $k_{i}$ is the degree of the node $i$. After $t$ time steps, a scale-free network with $m_{0}+t$ nodes and $m t$ edges will be generated.

The above information spread network is also the emotion diffusion network of the consensus system, each node equipped with an Agent who represents the individual in networks. Because each person has a recognition or evaluation on the status of society and these recognitions or evaluations are usually shown as emotions, we regard the individual $i$ 's emotion to the society as $x_{i}(t)$ at time $t$. Usually, the value of $x_{i}(t)$ can be discrete [26] $\left(x_{i}(t)= \pm 1\right)$ or continuous $[27,28]\left(-1 \leq x_{i}(t) \leq 1, x_{i}(t) \in R\right)$ in the study of consensus dynamics. Since continuous value is more suitable for reflecting the change process of individual emotions, we will take a continuous value for $x_{i}(t)$ in this paper.

In fact, in any society, all kinds of events are happening all the time. Some events attract more people's attention because of their specificity or sensitivity. And so the events change to hot events whose information spread very fast on the Internet. Because most of the hot events are full of "positive energy" or "negative energy," the process of information spread is also the process of emotion spread in the interpersonal relationship network. Usually, the information spread process of hot events contains two key subprocesses: the first subprocess is emotion diffusion. The core issue of this subprocess is whether the people who access the hot events are affected by it or will spread the information to other people. The second subprocess is emotion influence. That is, the process after the first subprocess (emotion diffusion) 
tends to be stable. People who have accessed the hot events change their emotions by discussion in this subprocess. We will propose the model of two subprocesses which are corresponding to the emotion diffusion mechanism and emotion influence mechanism in the following.

2.1. Emotion Diffusion Mechanism. All social consensus is generated from the social hot events and exposed by few people who we called sponsors of emotion spread, and they get an initial emotion whose value is $x_{0}\left(-1 \leq x_{0} \leq 1\right)$. The sponsor $j$ will pass the event information and negative emotions to his neighbor $i$ through the interpersonal relationship network. Now, the neighbor $i$ should face two problems.

One is the accepting degree of emotions passed by the sponsor. The accepting degree is usually determined by $i$ 's conformity (acceptability) $\alpha_{i}$ and the sponsor's impact $\beta_{j}$ [27]. The lager the individual conformity and the sponsor's impact are, the greater the individual accepting degree will be [2]. According to the literatures [2, 27], we assume that the emotion impact degree of the sponsor $j$ to neighbor $i$ is proportional to $x_{0}, \alpha_{i}$, and $\beta_{j}$. Therefore, at time $t+1$, the emotion of neighbor $i$ can be described as

$$
x_{i}(t+1)=x_{i}(t)+\alpha_{i} \beta_{j} x_{0},
$$

where $\alpha_{i} \sim U(0,1), \beta_{j} \sim U(0,1)$.

Another problem is whether the individual wants to transfer the emotions to others who do not access the hot event yet. In fact, whether the individual will continue to transfer the information depends on the degree of event influence and importance [29]. According to the literature $[29,30]$, we assume that the probability that individual continues to spread the negative emotions is

$$
P=\frac{1}{1+e^{-\alpha_{i} \beta_{j}\left|x_{0}\right|}},
$$

where $\left|x_{0}\right|$ is the initial emotion generated by the hot event, which represents the importance of the event. $\alpha_{i} \beta_{j}$ represents the influence of the negative emotions on individual.

Emotion diffusion mechanism shows that the individual's emotion will be affected by events in varying degrees. And probability that individuals spread the negative emotion increases with the influence of events. The most important characteristic of emotion diffusion process is the emotion spread and dissemination on the network, which is a scale expansion process of the individuals who contact hot events.

2.2. Emotion Influence Mechanism. Events information and emotions quickly spread by the emotion diffusion mechanism in Section 2.1 after a social hot event occurs. In general, the duration of the process is short, and the longer process is the continual interactions and discussion among the individuals after the information diffusion. Individual's emotion will be influenced by neighbor groups and so evolve dynamically in the process of exchange and discussion and even tend to group polarization [27].

In fact, there are two main factors that determine an individual's emotion during the process of emotion influence.
One is individual influence scope, and we use the proportion of individual degree of the total degree to measure this influence; for example, if individual $j$ is one of individual $i$ 's neighbors, the influence of individual $j$ on individual $i$ is determined by the ratio of $d_{j} / d_{i t}$, where $d_{j}$ is $j$ 's degree and $d_{i t}$ is the total degrees of all is neighbors. The other one is the differences of emotion. Because individuals always like to talk with individuals standing on the same camp but ignore the different views [28], if the emotions $x_{i}$ and $x_{j}$ differ by more than a fixed parameter $\varepsilon$, nothing happens because the two Agents think too differently to interact [31]. So the larger the differences in individual emotion, the smaller the mutual influence, and the converse is also true. Consistent with [31], we use $e^{-\left|x_{i}-x_{j}\right|}$ that represents the influence of emotion differences. Based on the above analysis, we define the influence of neighbor's emotion as

$$
I_{N i}=\frac{1}{n_{i}-1} \sum_{i=1}^{n_{i}} \frac{d_{j}}{d_{i t}} e^{-\left|x_{i}-x_{j}\right|} x_{j},
$$

where $x_{j}$ is the emotion of individual $j$ and $n_{i}$ is the neighbor number of $i . d_{j} / d_{i t}$ is the ratio of individual degree of total degree and its value determines the influence of $j$ on $i$.

According to the theory of social comparison [29], when $I_{i}>0$, influence of neighbor's emotion tends to be positive. Then, under this situation, it is possible for individual to tend to change emotion to positive, and the bigger $I_{i}$, the bigger the emotion changing probability. On the contrary, when $I_{i}<$ 0 , the influence of neighbor's emotion tends to be negative. Now, individual maybe tends to change the emotion to negative, and the smaller $I_{i}$, the bigger the emotion changing probability. So, we present the following conversion rules of emotion as [27].

For individual $i$, when $I_{i}>0$, add a small positive $\varepsilon(\varepsilon>0)$ on its emotion according to the probability $P_{1}=1 /\left(1+e^{-I}\right)$ :

$$
x_{i}(t+1)=x_{i}(t)+\varepsilon .
$$

On the contrary, when $I_{i}<0$, subtract a small positive $\varepsilon(\varepsilon>0)$ on its emotion according to the probability $P_{2}=$ $1 /\left(1+e^{I}\right)$ :

$$
x_{i}(t+1)=x_{i}(t)-\varepsilon .
$$

Over a period of time, hot events maybe arise several times, so the above process of emotion diffusion and influence will repeat continuously. Obviously, the average emotion of all individuals in system is one key factor which can represent the overall state of the social consensus system. Therefore, we define the parameter of system frangibility as

$$
r=\frac{1}{N} \sum_{i=1}^{N} x_{i}(t)
$$

Obviously, the social consensus system tends to be stable when $r \rightarrow 1$ and tends to be vulnerable when $r \rightarrow-1$.

The detailed simulation algorithm is as follows.

Step 1. Generate BA Network with $N$ nodes as the information spread network, every node represents one Agent, and initialize the parameters $\alpha_{i}, \beta_{j}$, and $\varepsilon$. 


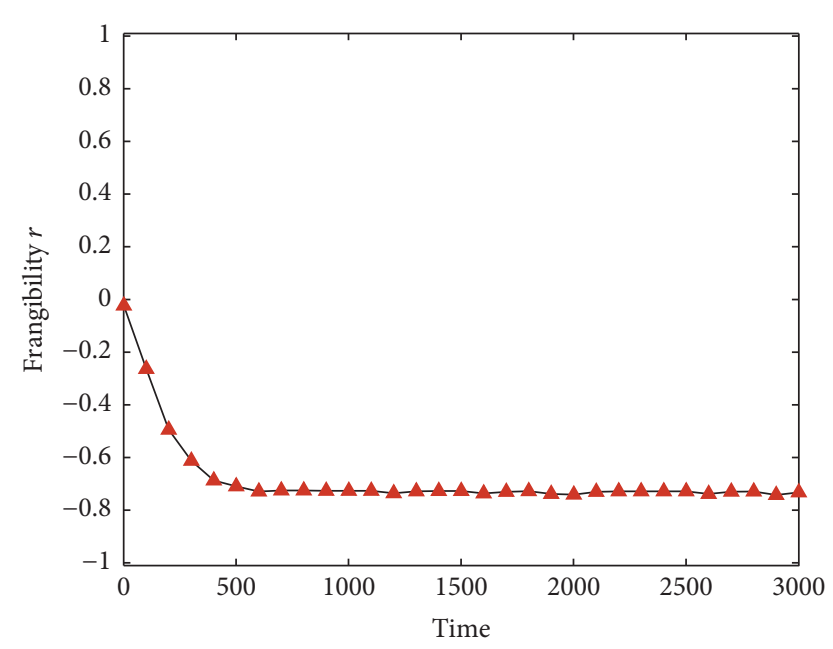

(a) Evolution of the frangibility in social consensus system
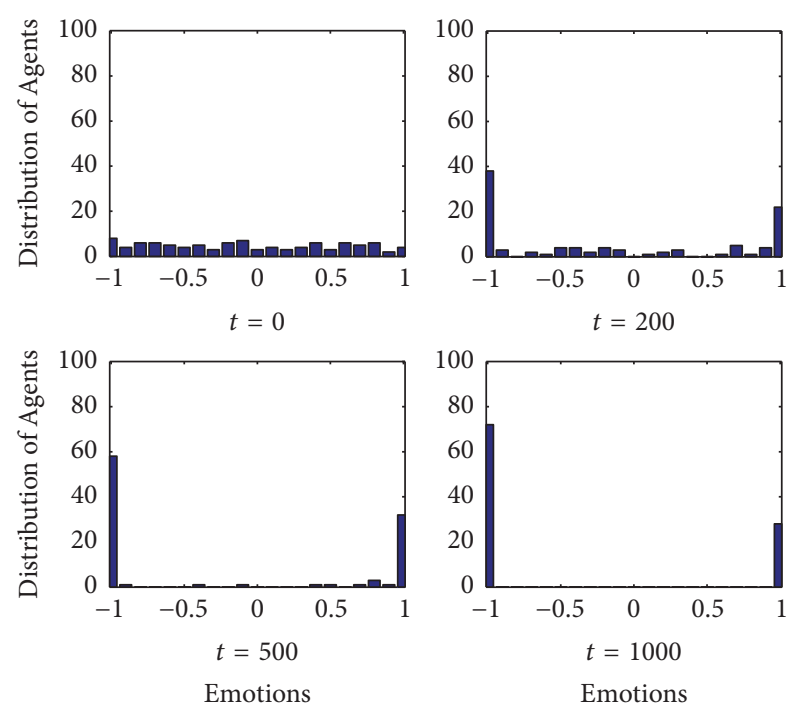

(b) Evolution of individual emotion distribution

FIGURE 1: Evolution of social consensus system with low negative event frequency.

Step 2. Select one node randomly as the negative emotion sponsor every time interval, and initialize $x_{0}$ between -1 and 0 as the initial value of the negative emotion.

Step 3. Update the value of emotion through emotion diffusion mechanism introduced in Section 2.1.

Step 4. Update the value of emotion through emotional influence mechanism introduced in Section 2.2.

Step 5. Repeat Steps 3 and 4 until the system tends to be stable.

The standard of system stability is that the emotions of individuals do not change anymore at the last 500 steps. When the system reaches stability, the simulation will terminate. It is important to note that the system frangibility is an important factor that affects the system evolution, and it will determine where the system terminates during the evolution sometimes [32,33]. In this paper, the system frangibility is comprehensively determined by negative event frequency, opinion leaders, and government shielding. We will reveal the complex dynamics in the next section.

\section{Simulation Results and Analysis}

Based on the simulation model established in Section 2, we generate a scale-free network where $N=100$. Each node is equipped with an Agent to represent an individual in the social consensus system. We take the initial state parameters of Agent are $\alpha_{i} \sim U(0,1), \beta_{j} \sim U(0,1), x_{0} \sim U(-1,0)$, and $\varepsilon=0.01$, and the initial emotion distribution of Agent follows a uniform distribution from -1 to 1 . It is worth emphasizing that different values of $\varepsilon$ may influence the evolutionary process of system. High values of $\varepsilon$ significantly accelerate the convergence process, and small $\varepsilon$ will take more time to achieve stability. The results of simulation are the average value of 30 experiments. We experiment for $N=200,300$, and 500, respectively, and get similar results except for the computation time.

3.1. Influence of Negative Event Frequency. Each negative event is associated with the spread of negative emotions. Firstly, we simulate the influence on social consensus system under the different frequencies of negative emotions spread (Figures 1 and 2). As we can see from Figures 1 and 2, individuals with positive emotions play an active role in the emotion influence process when the frequency of negative events is low (once every 50 simulation times). Although it has experienced the influence of negative emotions many times, frangibility in social consensus was still maintained at a certain level (Figure 1(a)). At this time, the group is divided into two extremes, the positive emotion group and the negative emotion group, and group polarization emerged (Figure 1(b)). With the increase of negative events frequency (once every 20 simulation times), system lost the support of the positive emotion group. Finally, social consensus system collapses completely (Figure 2 ). It is observed that the spread of high-frequency negative emotion can enhance the frangibility in social consensus than low-frequency one.

From 2010 to 2011, many countries in the Middle East have launched a series of large-scale collective behaviors called "Arab Spring" [3]. Among these collective behaviors, "1.25 Incident in Egypt" [3] is the most famous one for its large-scale, long-lasting, complete, and clear evolution process. So, we will take "1.25 Incident in Egypt" as an example to analyze the simulation results and reveal how the spread of negative emotions influences vulnerability of social consensus system as follows.

In fact, "Egypt 1.25 incident" is not an accident caused by extreme behavior but a breakout of the people's negative 


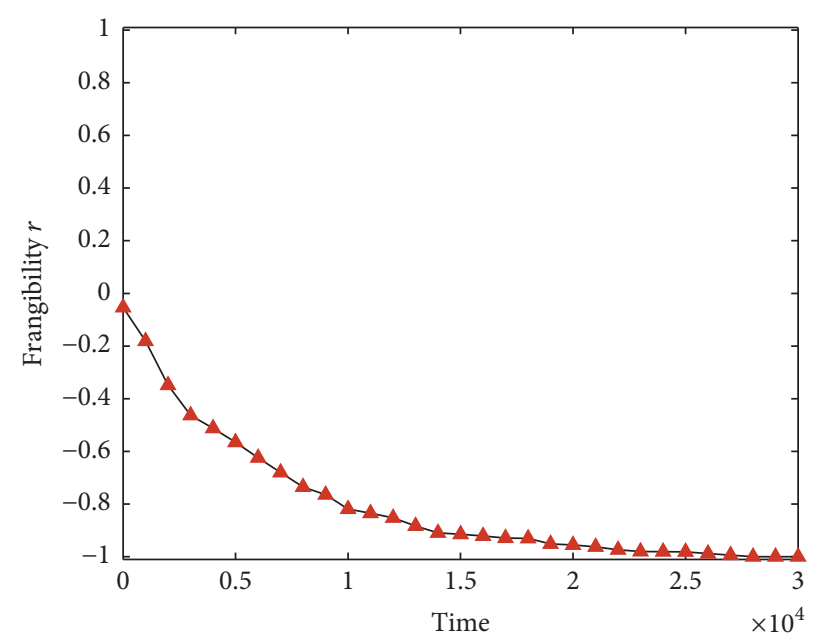

(a) Evolution of the frangibility in social consensus system

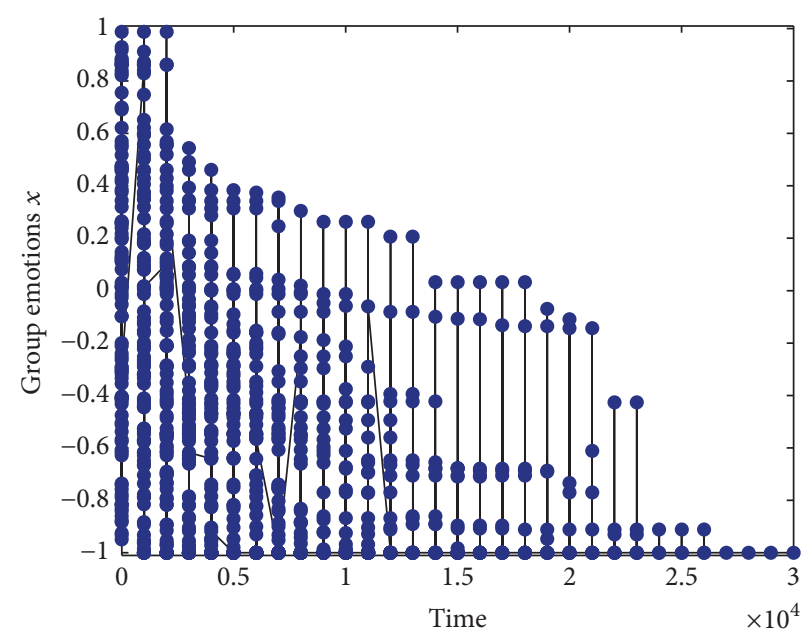

(b) Evolution of group emotion

FIGURE 2: Evolution of social consensus system with low negative event frequency.

emotions under the accumulation of negative events. Before the "Twitter Revolution" in Tunisian and the "Saïd Incident" [3], the Egyptian government has widespread problems of autocracy and corruption, negative events break out every now and then, and persons who live in the bottom society have been depressed for a long time. But, at this point, the diffusion frequency of negative emotions is relatively low, it has not infected the emotions of middle-class people completely, and negative emotions are not enough to make this part of people give up the positive support to the Egyptian government. Therefore, the social consensus system was still in part stable state at this time (Figure 1). However, after a long time of dictatorial regime of Mubarak, unemployment rate increased, police violence and official corruption occurred frequently, and negative events often happened, situation of society was turbulent, and negative emotions even began to spread in the middle-class people. After the Tunisian "Twitter Revolution" and "Saïd Incident," large-scale negative emotions caused by the "fuse" incident spread at a higher frequency and the social consensus system collapsed completely (Figure 2). The negative social events in Egypt from low frequency to high frequency have been going on for a long time, with the increase of frequency of negative events, frequency of negative emotions spread speeded up, and people used Twitter, Facebook, and other online community tools to spread the negative emotions spontaneously, which increase the vulnerability in social consensus system gradually and lead to the collapse of the system in the end.

3.2. Influence of Opinion Leaders. The negative event frequency impact on evolution of the frangibility in social consensus system is important, while a special type of individuals in the group usually tends to be ignored, that is, opinion leaders who usually emerge sometimes during the evolution of social consensus system. Though few researchers begin to care about the issue in recent years, they do not consider the new situation and new features of the Internet $[29,34]$. Here, according to the important role in evolution of Internet group emotion, we take the factor of opinion leaders into account. We will focus effort on how the opinion leaders impact on the frangibility in social consensus system as follows.

We randomly select one node as opinion leader of social consensus system in consensus information spread network at first. And, considering the stubbornness of opinion leader, we make its emotion always -1 [35]. By increasing the influence parameter of opinion leader $\beta$, we get the same conclusions as drawn in literature $[29,34-36]$. That is to say, the appearance of opinion leaders with negative emotion promotes the emergence of consensus and strengthens the frangibility of social consensus system (Figure 3 ).

As we can see from Figure 3(a), though public consensus system is impacted by the negative emotions constantly, the system still can maintain a certain frangibility level before the opinion leaders appearance. With the emergence of opinion leader with negative emotions, the balance of public consensus system is broken. Under the influence of opinion leader, the consensus system evolves rapidly and tends to be more vulnerable, which eventually steps into a system crash. Figure 3(b) shows that, with the influence of opinion leader increasing, the speed of frangibility of social consensus system tends to speed up.

Then, we will analyze the effects of opinion leaders in "Egypt 1.25 Incident." As opinion leaders, social activist Mashahed who is a well-known journalist that publishes images, pictures, and other information constantly through the video site, which causes more and more participants to join in the protest team. These opinion leaders have played a key role in the development of "Egypt 1.25 Incident." Before the opinion leaders appeared, the social consensus system had been affected by the negative emotions of the "Twitter Revolution" and "Saïd Incident," but the middle class do not completely go backward to toiler group, and 


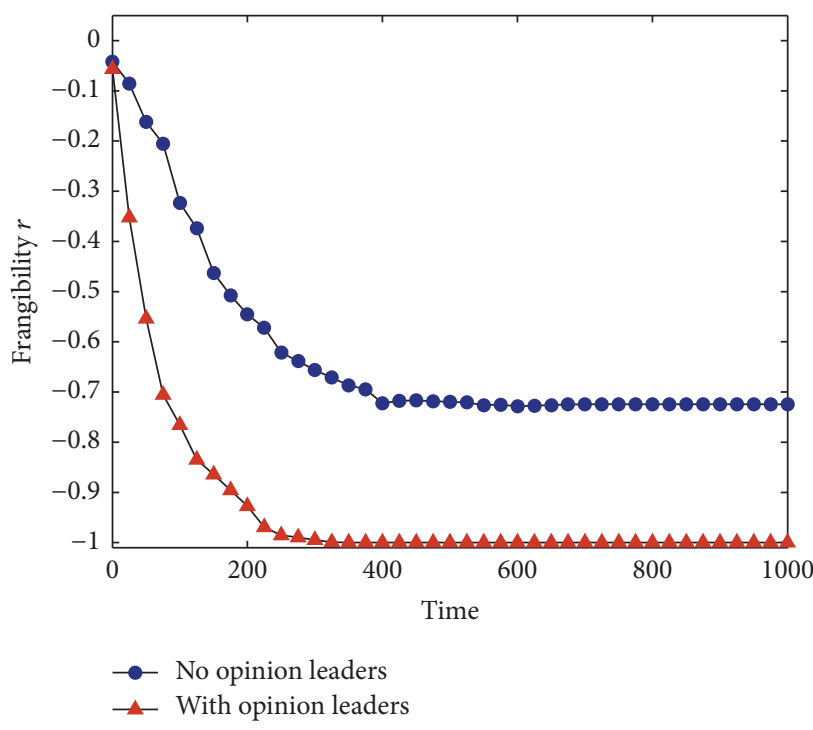

(a) Evolution of the system frangibility before and after the emergence of opinion leaders

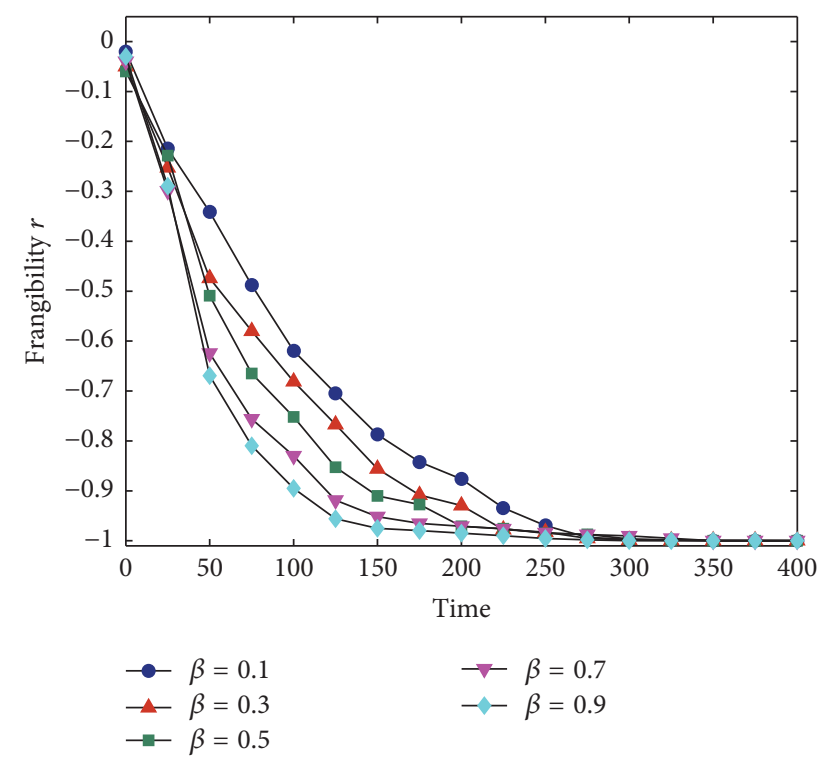

(b) Influence of opinion leaders on system frangibility under different $\beta$

FIGURE 3: Influence of opinion leaders on the frangibility in social consensus system.

the vulnerability of social consensus system still maintained a certain level (Figure 3(a)). With more and more opinion leaders such as the influential social activists and well-known journalists publicizing and disseminating information about the processions and rallies actively, the procession and protest team become bigger and bigger. Negative emotions spread to an unprecedented situation and finally the wavering middle class and even some upper-class also are pulled into the protest team. With the rapid development of the Internet and new media, popular websites and blogs have gradually developed into influential public opinion leaders. Their influence is determined by their authority and the number of their fans. The greater the influence is, the stronger the ability that the system evolves toward its own views will be (Figure 3(b)). The opinion leaders in "Egypt 1.25 Incident" are important influential members of the community, and their behavior leads Egyptian social consensus system to collapse.

3.3. Influence of Government's Shielding Behavior. In order to prevent the spread of negative emotions, the government might take technical measures like cutting Internet to shield the spread of information. So, we take the experiments on how the shielding behavior will influence the consensus system's frangibility as follows. We start the simulation system at first, and, then, when the simulation time reaches 1000 , we randomly remove 50 nodes to simulate the government's behavior of shielding Internet. Figure 4 shows the results of several simulation experiments. We can see that though the government has taken the shielding behaviors (after simulation experiments reach 1000), they still undergo failure to prevent the social consensus system to tend to be vulnerable. On the contrary, they make frangibility of social consensus system increased dramatically, and government's behavior

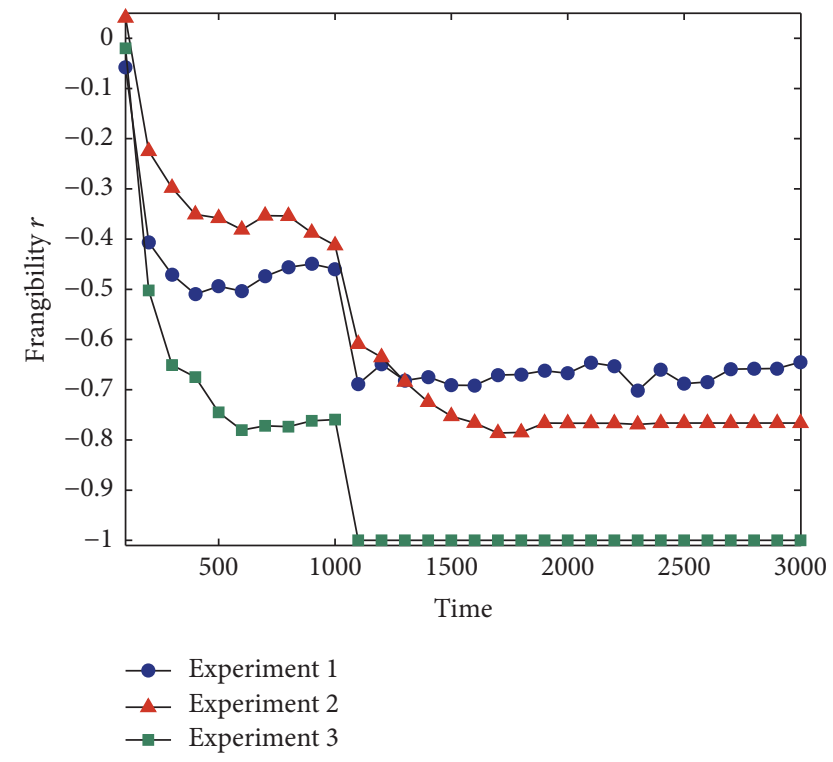

FIGURE 4: Evolution of the frangibility in social consensus system under government shielding behavior.

makes opposite effects. In fact, the government's information shielding behavior is a significant negative consensus event on itself, and at the same time it may lead to rapid spread of negative emotions among individuals, which will speed up the collapse of social consensus system in the end.

Finally, we analyze the effects of government's shielding behavior in the "Egypt 1.25 Incident." More than half of the people in Egypt are Internet users. Most of them have registered at Twitter, Facebook, and other online community 
tools. At the early time of the "Egypt 1.25 incident," people spread the negative emotions of the government through these online community tools, telephones, or other communication tools which provided a communication platform for the breakout of "Egypt 1.25 incident." In order to curb this phenomenon and control the spread of negative emotions, the Egyptian government blocks and interrupts the Internet and even telephone communication for a long time. However, people have a deep-rooted negative impression to the Egyptian government, and these measures not only fail to control consensus but also exacerbate the people's confrontation emotions to government. People consider the government's blocking behavior itself as a serious negative event and continue to spread information through Internet, mouth to mouth, and so on. Combining with the guidance of opinion leaders, negative emotions spread among the people and increase the vulnerability of social consensus system (Figure 4).

\section{Conclusions and Discussions}

Social consensus system is a complex system which is formed by the diffusion and spread of consensus through complex network of interpersonal relationships. Like the most complex systems [6-14], social consensus system also has the characteristic of frangibility. When the average value of the individual emotions in the system is negative, the system becomes vulnerable. Different from precious studies which adopt the methods of the evaluation index system $[5,15]$ or mathematical model $[16]$, this paper studies the evolutionary mechanism of frangibility in social consensus system based on Multiagent method. The simulation results show that high-frequency diffusion of negative emotion can enhance the frangibility of social consensus system compared to low-frequency one. The spread of high-frequency negative emotion even leads to system collapse and largescale collective behaviors. In order to reduce the risk of social consensus effectively, government should avoid negative incidents by improving public service awareness and preventing public relations crisis, and improving the level of emergency management, and the positive propagandas and consensus guidance also should be strengthened at the same time. In addition, opinion leaders play an important role in the evolution of social consensus frangibility. The negative emotions propagated by opinion leaders may spread widely and fast, shielding behavior could not inhibit the spread of negative emotions; on the contrary, it will strengthen the frangibility of consensus system. So, the government should guide the opinion leaders correctly, for example, paying more attentions to deal with the negative events exposed by opinion leaders in time so as to prevent the rapid spread of negative emotions. Another suggestion is that the government can develop a government image management system based on the network data technology to realize the effective monitoring and early warning of the network consensus, so as to eliminate the negative impact of events and maintain social stability.

As the social consensus system is a typical self-organized emerging system which has the micro-macro effects, the characteristics of Multiagent System determine that it is not only a powerful tool to explore the micromechanism of complex systems but also an important method to study the micro-macro effects [37], which is very suitable to study the evolution of complex system [17]. Simulation results show that Multiagent simulation model proposed in this paper can reveal the evolution mechanism and provide a new perspective for the research of the frangibility in social system. Many further studies may be developed as the following aspects: (1) building a network model based on the actual data through the analysis of social network. Analyzing the impacts of network structure, individual characteristics, and negative events on the frangibility in social consensus system. (2) Studying the evolution of frangibility in social consensus system in the process of negative emotion interaction between online and offline; (3) using big data technology for analysis of negative emotion diffusion in social consensus system based on typical cases.

\section{Conflicts of Interest}

The authors declare that there are no conflicts of interest regarding the publication of this article.

\section{Acknowledgments}

This research was supported by the National Statistical Science Foundation of China (Grant no. 2016LZ20), National Natural Science Foundation of China (Grant nos. 71671060, 41501183), Natural Science Foundation of Hubei (Grant no. 2014CFB374), and Science and Technology Innovative Team Foundation of Hubei (Grant no. T201516).

\section{References}

[1] J. Olick K, V. Vinitzky-Seroussi, and D. Levy, Eds., The Collective Memory Reader, Oxford University Press, New York, NY, USA, 2011.

[2] Y. Zhang and R. Xiao, "Modeling and simulation of synchronous threshold in vent collective behavior," Discrete Dynamics in Nature and Society. An International Multidisciplinary Research and Review Journal, no. atricle 170968, 12 pages, 2014.

[3] E. D. Mansfield and J. Snyder, "Democratization and the Arab Spring," International Interactions: Empirical and Theoretical Research in International Relations, vol. 38, no. 5, pp. 722-733, 2012.

[4] N. R. Kleinfield and C. Buckley, Wall Street Occupiers, Protesting till Whenever. New York times, 2011, October 1, A1.

[5] L. Rygel, D. O'Sullivan, and B. Yarnal, "A method for constructing a social vulnerability index: an application to hurricane storm surges in a developed country," Mitigation and Adaptation Strategies for Global Change, vol. 11, no. 3, pp. 741-764, 2006.

[6] A. Fekete, M. Damm, and J. Birkmann, "Scales as a challenge for vulnerability assessment," Natural Hazards, vol. 55, no. 3, pp. 729-747, 2010.

[7] H.-M. Füssel, "How inequitable is the global distribution of responsibility, capability, and vulnerability to climate change: a comprehensive indicator-based assessment," Global Environmental Change, vol. 20, no. 4, pp. 597-611, 2010. 
[8] C. M. Beier, T. M. Patterson, and F. S. Chapin III, "Ecosystem services and emergent vulnerability in managed ecosystems: A geospatial decision-support tool," Ecosystems, vol. 11, no. 6, pp. 923-938, 2008.

[9] D. R. Pathak and A. Hiratsuka, "An integrated GIS based fuzzy pattern recognition model to compute groundwater vulnerability index for decision making," Journal of Hydro-Environment Research, vol. 5, no. 1, pp. 63-77, 2011.

[10] K. J. Farn and S. K. Lin, "A study on information security management system evaluation-assets, threat and frangibility", Computer Standards and Interfaces, vol. 26, no. 6, pp. 501-513, 2004.

[11] P. Holme, B. J. Kim, C. N. Yoon, and S. K. Han, "Attack vulnerability of complex networks," Physical Review E, Statistical, Nonlinear, and Soft Matter Physics, vol. 65, no. 5, article 056109, 2002.

[12] M. E. J. Newman, S. Forrest, and J. Balthrop, "Email networks and the spread of computer viruses," Physical Review E, vol. 66, no. 3, article 035101, 2002.

[13] D. Magoni, "Tearing down the Internet," IEEE Journal on Selected Areas in Communications, vol. 21, no. 6, pp. 949-960, 2003.

[14] M. Ouyang, L. Hong, Z.-J. Mao, M.-H. Yu, and F. Qi, "A methodological approach to analyze vulnerability of interdependent infrastructures," Simulation Modelling Practice and Theory, vol. 17, no. 5, pp. 817-828, 2009.

[15] W. N. Adger, N. Brooks, G. Bentham, M. Agnew, and S. Eriksen, "New indicators of frangibility and adaptive capacity [R]," Tech. Rep. 7, Tyndall Centre for Climate Change Research, 2004.

[16] B. Dixon, "Groundwater vulnerability mapping: a GIS and fuzzy rule based integrated tool," Applied Geography, vol. 25, no. 4, pp. 327-347, 2005.

[17] R. Xiao, Y. Zhang, and Z. Huang, "Emergent computation of complex systems: A comprehensive review," International Journal of Bio-Inspired Computation, vol. 7, no. 2, pp. 75-97, 2015.

[18] G. Lu and J. Lu, "Introduction to the investigating in neural trust and multi agent systems," in Examining Information Retrieval and Image Processing Paradigms in Multidisciplinary Contexts, J. Lu and Q. Xu, Eds., pp. 269-273, IGI Global, Hershey, Pa, USA, 2017.

[19] D. Rosaci, G. M. L. Sarné, and S. Garruzzo, "Integrating trust measures in multiagent systems," International Journal of Intelligent Systems, vol. 27, no. 1, pp. 1-15, 2012.

[20] T. D. Huynh, N. R. Jennings, and N. R. Shadbolt, "An integrated trust and reputation model for open multi-agent systems," Autonomous Agents and Multi-Agent Systems, vol. 13, no. 2, pp. 119-154, 2006.

[21] D. Rosaci, “Trust measures for competitive agents," KnowledgeBased Systems, vol. 28, pp. 38-46, 2012.

[22] M. Wooldridge, An Introduction to Multi-Agent Systems, John Wiley \& Sons, New York, NY, USA, 2009.

[23] H. P. Thadakamalla, U. N. Raghavan, S. Kumara, and R. Albert, "Survivability of multiagent-based supply networks: a topological perspective," IEEE Intelligent Systems, vol. 19, no. 5, pp. 24-31, 2004.

[24] A. Nair and J. M. Vidal, "Supply network topology and robustness against disruptions - An investigation using multi-agent model," International Journal of Production Research, vol. 49, no. 5, pp. 1391-1404, 2011.
[25] A. L. Barabási and R. Albert, "Emergence of scaling in random networks," American Association for the Advancement of Science. Science, vol. 286, no. 5439, pp. 509-512, 1999.

[26] D. Stauffer, "Difficulty for consensus in simultaneous opinion formation of Sznajd model," Journal of Mathematical Sociology, vol. 28, no. 1, pp. 25-33, 2004.

[27] Y. Zhang and R. Xiao, "Modeling and simulation of polarization in internet group opinions based on cellular automata," Discrete Dynamics in Nature and Society, vol. 2015, Article ID 140984, 14 pages, 2015.

[28] G. Deffuant, D. Neau, F. Amblard, and G. Weisbuch, "Mixing beliefs among interacting agents," Advances in Complex Systems, vol. 3, pp. 87-98, 2000.

[29] J. A. Holyst, K. Kacperski, and F. Schweitzer, "Phase transitions in social impact models of opinion formation," Physica A, vol. 285, no. 1-2, pp. 199-210, 2000.

[30] G. Szabó and G. Fáth, "Evolutionary games on graphs," Physics Reports. A Review Section of Physics Letters, vol. 446, no. 4-6, pp. 97-216, 2007.

[31] A. Pluchino, V. Latora, and A. Rapisarda, "Changing opinions in a changing world: a new perspective in sociophysics," International Journal of Modern Physics C, vol. 16, no. 4, pp. 515531, 2005.

[32] A. Szolnoki, Z. Wang, and M. Perc, "Wisdom of groups promotes cooperation in evolutionary social dilemmas," Scientific Reports, vol. 2, article 576, 2012.

[33] H. Rauhut and J. Lorenz, "The wisdom of crowds in one mind: how individuals can simulate the knowledge of diverse societies to reach better decisions," Journal of Mathematical Psychology, vol. 55, no. 2, pp. 191-197, 2011.

[34] M. Perc, A. Szolnoki, and G. Szabó, "Restricted connections among distinguished players support cooperation," Physical Review E-Statistical, Nonlinear, and Soft Matter Physics, vol. 78, no. 6, Article ID 066101, 2008.

[35] M. Mobilia, "Does a single zealot affect an infinite group of voters?” Physical Review Letters, vol. 91, article 028701, no. 2, 2003.

[36] A. Szolnoki, M. Perc, and M. Mobilia, "Facilitators on networks reveal optimal interplay between information exchange and reciprocity," Physical Review E, vol. 89, no. 4, Article ID 042802, 2014.

[37] F. Zambonelli and A. Omicini, "Challenges and research directions in agent-oriented software engineering," Autonomous Agents and Multi-Agent Systems, vol. 9, no. 3, pp. 253-283, 2004. 


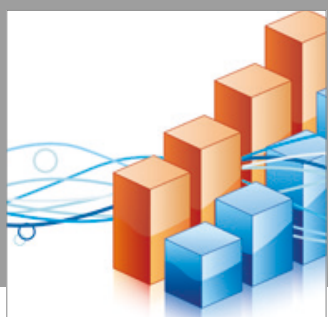

Advances in

Operations Research

vatersals

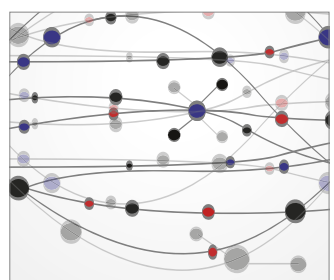

\section{The Scientific} World Journal
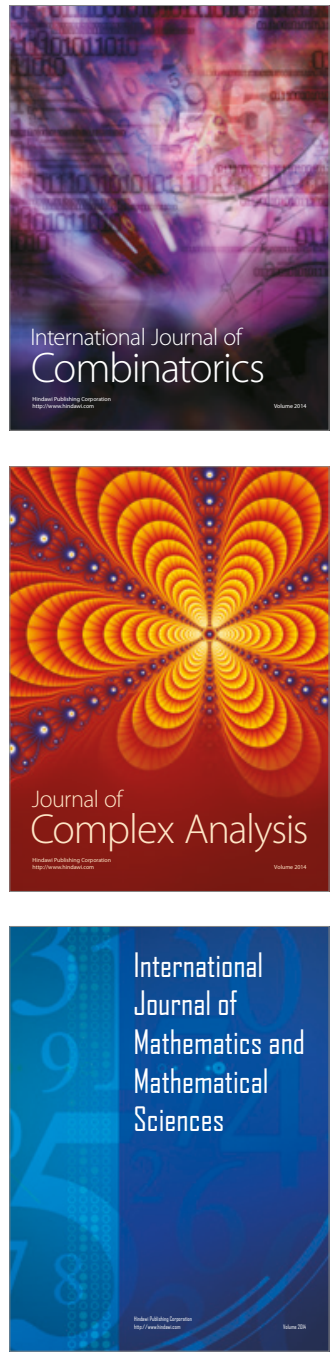
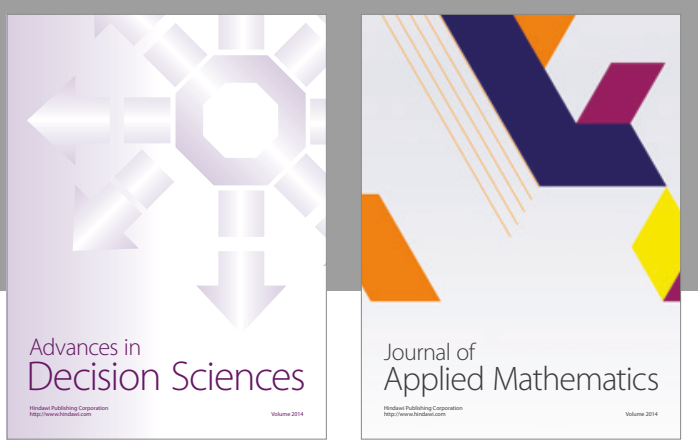

Algebra

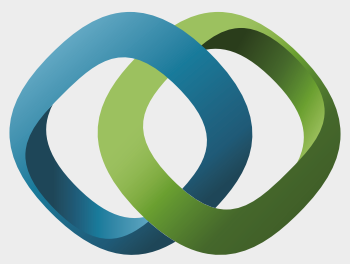

\section{Hindawi}

Submit your manuscripts at

https://www.hindawi.com
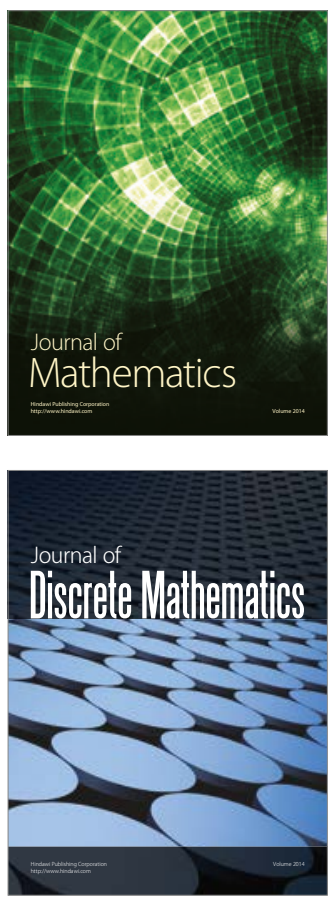

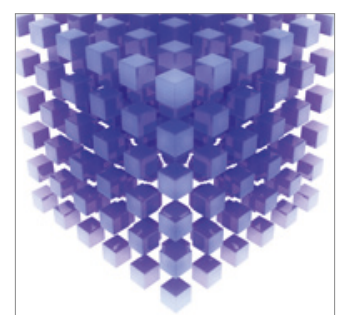

Mathematical Problems in Engineering
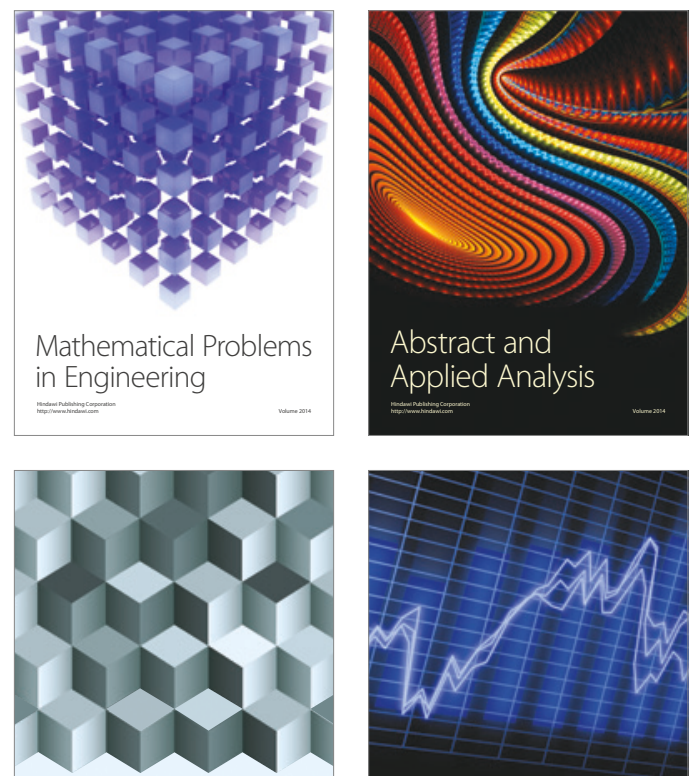

Journal of

Function Spaces

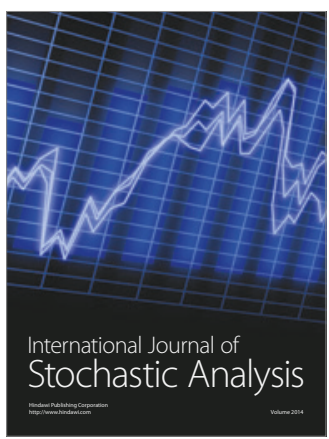

Probability and Statistics
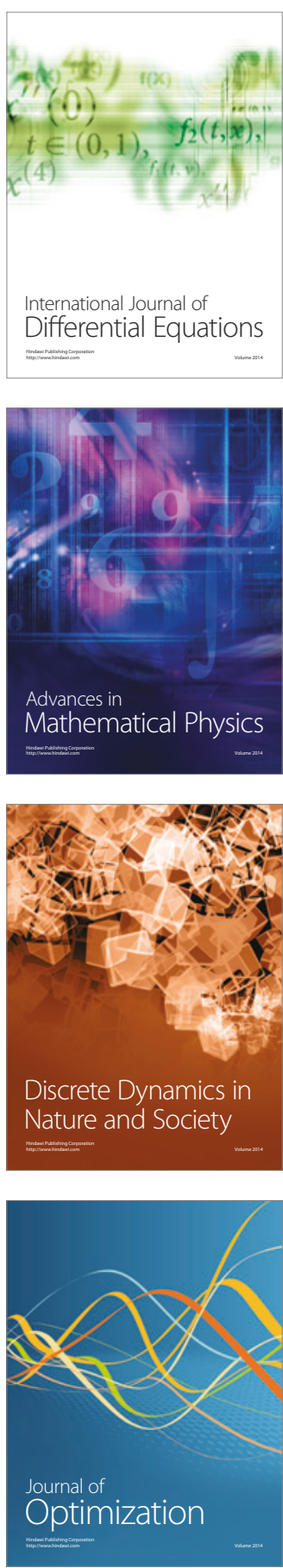